\title{
SEMI-INVEX FUNCTIONS AND THEIR SUBDIFFERENTIALS
}

\author{
J. Dutta, V. Vetrivel and S. NANDA
}

\begin{abstract}
We introduce the notion of semi-invex function (non-smooth) and the associated subdifferential. We study their properties and establish the conditions for optimality in constrained and unconstrained minimisation problems.
\end{abstract}

\section{INTRODUCTION}

The theory of subdifferentials for locally Lipschitz functions due to Clarke [1] has been well developed in the recent past. In this paper we introduce subdifferentials for functions not necessarily locally Lipschitz. We study their properties and establish the conditions for optimality in constrained and unconstrained minimisation problems.

We start with the notion of invex functions, introduced by Hanson [3]. A differentiable function $f: R^{n} \rightarrow R$ is said to be invex with respect to a given $\eta: R^{n} \times R^{n} \rightarrow R^{n}$ if for any $x, y \in R^{n}$

$$
f(y)-f(x) \geqslant\langle\nabla f(x), \eta(y, x)\rangle
$$

where $\nabla f(x)$ is the gradient vector of $f$ at $x$ and $\langle.,$.$\rangle denotes the usual inner product$ of vectors. The importance of this class of functions lies in the fact that for the constrained optimisation problems the Karush-Kuhn-Tucker conditions are sufficient for optimality if the objective and constraint functions are invex with the same $\eta$, thereby relaxing the need for convexity in such a case.

We observe that $\nabla f(x)$ is not the only element in $R^{n}$ which satisfies (1) with the given $\eta$. For example if $f: R \rightarrow R$ is a constant function and $\eta(y, x)=(y-x)^{2}$ then any $x^{*} \in R$ such that $x^{*} \leqslant 0$ satisfies the following inequality:

$$
f(y)-f(x) \geqslant\left\langle x^{*}, \eta(y, x)\right\rangle
$$

This observation motivates us to introduce the following.

Received 9th December, 1996

The authors are grateful to Professor B.D. Craven of Melbourne University, for his valuable suggestions to improve the earlier version of the paper.

Copyright Clearance Centre, Inc. Serial-fee code: 0004-9729/97 \$A2.00+0.00. 
Definition 1.1: A function $f: R^{n} \rightarrow R$ is said to be semi-invex at $x \in R^{n}$ with respect to a given $\eta: R^{n} \times R^{n} \rightarrow R^{n}$ if for all $y \in R^{n}$ there exists $\xi \in R^{n}$ such that

$$
f(y)-f(x) \geqslant\langle\xi, \eta(y, x)\rangle
$$

We call such $\xi$, an $\eta$-subgradient of $f$ at $x$ and the set

$$
\partial^{\eta} f(x)=\left\{\xi \in R^{n}: f(y)-f(x) \geqslant\langle\xi, \eta(y, x)\rangle \text { for all } y \in R^{n}\right\}
$$

the $\eta$-subdifferential of $f$ at $x$. Therefore a function is semi-invex at a given point if it has a non-empty $\eta$-differential at that point. The function $f$ is said to be semi-invex if it is semi-invex at each $x \in R^{n}$. We note that the set $\partial^{\eta} f(x)$ is not necessarily singleton even if $f$ is differentiable and that it coincides with the subdifferential of convex analysis [7] when $f$ is a convex function and $\eta(y, x)=y-x$. Clearly the class of semi-invex functions includes the invex functions.

It is interesting to note that if $f: R^{n} \rightarrow R$ is semi-invex at $x \in R^{n}$ with respect to $\eta: R^{n} \times R^{n} \rightarrow R^{n}$ and $\psi: R \rightarrow R$ is a strictly increasing convex function, then $\psi \circ f$ is semi-invex at $x \in R^{n}$ with the same $\eta$. Yang and Craven [8] have also introduced the notion of $\eta$-subgradient and $\eta$-subdifferential for locally Lipschitz functions and established necessary conditions for optimality of constrained non-smooth programs involving the $\eta$-subdifferential.

Example 1.1. Consider the function $f: R \rightarrow R$ defined by $f(x)=-|x|$. $f$ is clearly semi-invex at $x=0$ with $\eta(y, x)=-(|y|+|x|)$. Here $\partial^{n} f(0)=[+1, \infty)$. We note that Clarke's subdifferential $[\mathbf{1}]$ at 0 is $[-1,+1]$ and this shows that in general the two subdifferentials are different.

Though Yang and Craven [8] have defined the $\eta$-subdifferential for locally Lipschitz functions, the following example which is essentially due to Yang and Craven [8] shows that a function can have a non-empty $\eta$-subdifferential at a point where it is not locally Lipschitz.

EXAMPLE 1.2. Let $f: R \rightarrow R$ and $\eta(x, y)$ be as follows:

$$
\begin{aligned}
& f(x)=\left\{\begin{array}{lll}
0 & : & x \leqslant 0 \\
\frac{1}{x} & : & x>0
\end{array}\right. \\
& \eta(x, y)=\left\{\begin{array}{lll}
y-x & : & x>0 \\
x-y & : & x<0 \\
0 & : & x=0
\end{array}\right.
\end{aligned}
$$

The function $f$ is not locally Lipschitz at $x=0$ since it is discontinuous there but still we have $\partial^{n} f(0)=\{v: v \geqslant 0\}$. Thus one can compute $\eta$-subdifferentials for 
functions which are not locally Lipschitz. Yang and Craven [8] also showed that for a class of locally Lipschitz functions called generalised invex introduced by Craven [2] the $\eta$-subdifferential remains non-empty. Let us recall that a function $f: R^{n} \rightarrow R$ is called generalised invex at $x \in R^{n}$ for a given $\eta$, if it is locally Lipschitz and satisfies

$$
f(y)-f(x) \geqslant\langle\xi, \eta(y, x)\rangle
$$

for all $\xi \in \partial f(x)$ and $x \in R^{n}$, where $\partial f(x)$ denotes the Clarke subdifferential at $x \in R^{n}$. (See Clarke [1].)

Further, Yang and Craven [8] have defined the $\eta$-generalised directional derivative $f_{\eta}^{\circ}(x, h)$ of $f$ at $x$ in the direction $h$ as the support function of the $\eta$-subdifferential when it is non-empty. More precisely

$$
f_{\eta}^{\circ}(x, h)=\sup \left\{\langle v, h\rangle: v \in \partial^{\eta} f(x)\right\}
$$

if $\partial^{\eta} f(x) \neq \emptyset$. If $\partial^{\eta} f(x)=\emptyset$ then we write $f_{\eta}^{\circ}(x, h)=-\infty$. If $v \in \partial^{\eta} f(x)$, then $f_{\eta}^{\circ}(x, h) \geqslant\langle v, h\rangle$ for all $x \in R^{n}$. The converse problem remains open.

\section{Properties of $\eta$-Subdifferentials}

The proof of the following proposition is straightforward and hence omitted.

Proposition 2.1. If $f: R^{n} \rightarrow R$ is semi-invex with $\eta: R^{n} \times R^{n} \rightarrow R^{n}$ then $\partial^{n} f(x)$ is a non-empty, closed and convex subset of $R^{n}$.

We note that $\partial^{n} f(x)$ is not compact in general. See Example 1.1. However we have

Proposition 2.2. Let $f: R^{n} \rightarrow R$ be semi-invex with $\eta: R^{n} \times R^{n} \rightarrow R^{n}$ such that $\eta$ is an open map and $\eta(x, x)=0$ for all $x \in R^{n}$. Let $x_{0} \in R^{n}$. If there exists a neighbourhood $U$ of $x_{0}$, such that

$$
|f(y)-f(x)| \leqslant K\|\eta(y, x)\|
$$

where $K>0$, then $\partial^{n} f(x)$ is compact.

Proof: If $x \in U$ and $\xi \in \partial^{n} f(x)$, then for all $y \in U$

$$
\langle\xi, \eta(y, x)\rangle \leqslant f(y)-f(x) \leqslant K\|\eta(y, x)\| .
$$

Since $\eta(x, x)=0$ and $\eta$ is an open map, the map $y \rightarrow \eta(y, x)$ maps $U$ onto an open neighbourhood $V$ of the origin. Thus at each $x \in U$ and $\xi \in \partial^{n} f(x)$

$$
\langle\xi, z\rangle \leqslant K\|z\|
$$

holds for all $z \in B(0, \varepsilon) \subseteq V$, where $B(0, \varepsilon)$ denotes the open ball centred at 0 with radius $\varepsilon$. Hence it follows that

$$
|\langle\xi, z\rangle| \leqslant K\|z\|
$$

which implies that $\|\xi\| \leqslant K$. Thus the map $x \rightarrow \partial^{n} f(x)$ is locally bounded at $x_{0}$ and hence $\partial^{n} f\left(x_{0}\right)$ is compact. 
REMARK. If $f$ is semi-invex with an arbitrary $\eta$ such that $f_{\eta}^{\circ}(x, h)$ is finite for all $h$, then it is easy to see that $\partial^{n} f(x)$ is compact if and only if

$$
f_{\eta}^{\circ}(x, h) \leqslant K\|h\| \quad \text { for all } h \in R^{n}
$$

for some $K>0$. The following example due to Yang and Craven [8] illustrates the above results.

ExAMPLE 2.1. Let $f: R \rightarrow R$ and $\eta$ be as follows

$$
\begin{aligned}
& f(x)= \begin{cases}\frac{1}{2} x & : \quad x \geqslant 0 \\
x & : \quad x<0\end{cases} \\
& \eta(x, y)=\left\{\begin{array}{lll}
\frac{1}{2}(x-y) & : \quad x \geqslant 0 \\
x-y & : \quad x<0 .
\end{array}\right.
\end{aligned}
$$

Here $f$ and $\eta$ satisfy the hypothesis of the above Proposition and also (11) at $x=0$ and $\partial^{n} f(x)=\{1\}$.

Proposition 2.3. If $f: R^{n} \rightarrow R$ and $g: R^{n} \rightarrow R$ are semi-invex with the same $\eta$ so is $f+g$ and for each $x \in R^{n}$,

$$
\partial^{n} f(x)+\partial^{n} g(x) \subseteq \partial^{n}(f+g)(x)
$$

The proof is straight forward. We note that equality does not hold in general.

EXAmPLE 2.2. Let $f: R \rightarrow R$ and $g: R \rightarrow R$ be defined by

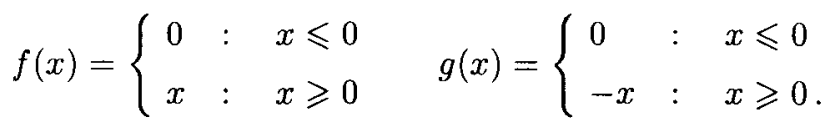

These functions are semi-invex with $\eta(y, x)=|y-x|$, and $\partial^{n} f(0)+\partial^{n} g(0)$ $=\{x: x \leqslant-1\}$ and $\partial^{n}(f+g)(0)=\{x: x \leqslant 0\}$.

We now recall that a function $f: R^{n} \rightarrow R$ is pre-invex with respect to a given $\eta: R^{n} \times R^{n} \rightarrow R^{n}$ if for all $x, y \in R^{n}$,

$$
f(x+\lambda \eta(y, x)) \leqslant \lambda f(y)+(1-\lambda) f(x)
$$

where $\lambda \in(0,1)$. Pini [5] showed that if $f$ is differentiable pre-invex, then it is invex. Recently Mohan and Neogi [4] have shown that the converse holds provided $\eta$ satisfies the following 
Condition C. A function $\eta: R^{n} \times R^{n} \rightarrow R^{n}$ is said to satisfy Condition C if for any $x, y \in R^{n}$ and $\lambda \in[0,1]$

(i) $\eta(x, x+\lambda \eta(y, x))=-\lambda \eta(y, x)$.

(ii) $\eta(y, x+\lambda \eta(y, x))=(1-\lambda) \eta(y, x)$.

Mohan and Neogi [4] have also showed that Condition $\mathrm{C}$ holds also for $\eta$ other than the trivial $\eta(y, x)=y-x$.

Proposition 2.4. If $f: R^{n} \rightarrow R$ is semi-invex with a given $\eta$ satisfying Condition $C$, then $f$ is pre-invex with the same $\eta$.

Proof: Since $f$ is semi-invex, there exists $\xi \in R^{n}$ such that for all $\lambda \in(0,1)$

$$
\begin{aligned}
& f(y) \geqslant f(x+\lambda \eta(y, x))+\langle\xi, \eta(y, x+\lambda n(y, x))\rangle \\
& f(x) \geqslant f(x+\lambda \eta(y, x))+\langle\xi, \eta(x, x+\lambda \eta(y, x))\rangle .
\end{aligned}
$$

By noting the fact that $\eta$ satisfies Condition $\mathrm{C}$, we have

$$
\begin{aligned}
& f(y) \geqslant f(x+\lambda \eta(y, x))+(1-\lambda)\langle\xi, \eta(y, x)\rangle \\
& f(x) \geqslant f(x+\lambda \eta(y, x))-\lambda\langle\xi, \eta(y, x)\rangle .
\end{aligned}
$$

By multiplying both sides of (14) and (15) by $\lambda$ and $(1-\lambda)$ and then adding we get

$$
f(x+\lambda \eta(y, x)) \leqslant \lambda f(y)+(1-\lambda) f(x) .
$$

Now the natural question is: 'Under what conditions will a pre-invex function be semi-invex?' This remains open.

It is interesting to note that even if $f: R^{n} \rightarrow R$ is a differentiable semi-invex function the gradient need not belong to the $\eta$-subdifferential. The following example illustrates this fact.

EXAmple 2.3. Consider $f: R \rightarrow R$ defined by $f(x)=x^{2}$. This function is a semiinvex function with respect to $\eta(y, x)=x^{2}$ for all $y \in R$, since $-1 \in \partial^{n} f(x)$ for any $x \in R$. Here $2 \notin \partial^{\eta} f(1)$.

We shall now present a sufficient condition for an element of $R^{n}$ to be an element of the $\eta$-subdifferential of a differentiable semi-invex function. For this we need the following definition due to Yang and Craven [8].

Definition 2.1: Let $S \subseteq R^{n}$ and $\eta: R^{n} \times R^{n} \rightarrow R^{n}$. The $\eta$-normal cone $N_{S}^{\eta}(x)$ at $x \in S$ is defined as

$$
N_{S}^{\eta}(x)=\left\{\xi \in R^{n}:\langle\xi, \eta(y, x)\rangle \leqslant 0 \quad \text { for all } y \in S\right\} .
$$


REMARK. It is clear that $0 \in N_{S}^{\eta}(x)$. If $S=R^{n}$ the $\eta$-normal cone need not be a singleton set containing the zero element alone, unlike the usual normal cone in convex analysis. For example, if $S=R$ and $\eta(y, x)=(y-x)^{2}$, then $N_{S}^{\eta}(x)=\{\xi \in R: \xi \leqslant 0\}$.

PRoposition 2.5. Let $f: R^{n} \rightarrow R$ be a differentiable semi-invex function with $\eta$ satisfying Condition C. Let $\xi \in R^{n}$. If

$$
\xi-\nabla f(x) \in N_{R^{n}}^{\eta}(x)
$$

then $\xi \in \partial^{\eta} f(x)$.

ProOF: Let $\xi-\nabla f(x) \in N_{R^{n}}^{\eta}(x)$. Then

$$
\langle\xi-\nabla f(x), \eta(y, x)\rangle \leqslant 0 \text { for all } y \in R^{n} \text {. }
$$

That is,

$$
\langle\nabla f(x), \eta(y, x)\rangle \geqslant\langle\xi, \eta(y, x)\rangle .
$$

Now from the Taylor's theorem we have

$$
f(x+\lambda \eta(y, x))=f(x)+\langle\nabla f(x), \lambda \eta(y, x)\rangle+o(\|\lambda \eta(y, x)\|) .
$$

By the above inequality,

$$
f(x+\lambda \eta(y, x)) \geqslant f(x)+\langle\xi, \lambda \eta(y, x)\rangle+o(\lambda\|\eta(y, x)\|) .
$$

Since $f$ is semi-invex with $\eta$ satisfying Condition $C$, by Proposition 2.4 we know that $f$ is pre-invex with the same $\eta$. Hence we have

$$
\lambda f(y)+(1-\lambda) f(x) \geqslant f(x)+\lambda\langle\xi, \eta(y, x)\rangle+o(\lambda\|\eta(y, x)\|) .
$$

Dividing by $\lambda$ and letting $\lambda \rightarrow 0+$, we get

$$
f(y)-f(x) \geqslant\langle\xi, \eta(y, x)\rangle .
$$

Since $y$ is arbitrary, $\xi \in \partial^{\eta} f(x)$.

REMARK. Note that by the above theorem $\nabla f(x) \in \partial^{\eta} f(x)$, since $\nabla f(x)-\nabla f(x)=$ $0 \in N_{R^{n}}^{\eta}(x)$. Hence every differentiable semi-invex function with $\eta$ satisfying Condition $\mathrm{C}$ is invex.

Assumption A. (Yang and Craven [8].) A locally Lipschitz function $f: R^{n} \rightarrow R$ is said to satisfy Assumption $\mathrm{A}$ at $x_{0} \in R^{n}$ if it is semi-invex at $x_{0}$ and the following holds:

$$
\limsup _{t-0+} \frac{f\left(x_{0}+t h\right)-f\left(x_{0}\right)}{t} \leqslant f_{\eta}^{\circ}\left(x_{o}, h\right) .
$$

Proposition 2.6. Let $f: R^{n} \rightarrow R$ be a locally Lipschitz, semi-invex function with $\eta$ satisfying Condition $C$. Let $x_{0} \in R^{n}$ be such that equality holds in (19). Then $f$ satisfies the generalised invex condition of Yang and Craven [8], at $x_{0}$, that is,

$$
f(x)-f\left(x_{0}\right) \geqslant f_{\eta}^{\circ}\left(x, \eta\left(x, x_{0}\right)\right) \text { for all } x \in R^{n} .
$$

The proof follows easily from Proposition 2.4 . 


\section{OPtimisation With SEMI-INVEX FUnCTIONS}

If $f: R^{n} \rightarrow R$ is semi-invex at $x_{0}$, a global minimum of $f$, then $0 \in \partial^{\eta} f\left(x_{0}\right)$ is both a necessary and a sufficient condition for optimality. But if $x_{0}$ is a local minimum, then it is not necessary that $0 \in \partial^{\eta} f\left(x_{0}\right)$. The following example illustrates this fact.

EXAMPLE 3.1. Let $f: R \rightarrow R$ be defined as follows:

$$
f(x)=\left\{\begin{array}{lll}
-\frac{1}{2} & : & \infty<x<\frac{1}{2} \\
x & : & -\frac{1}{2} \leqslant x<\frac{1}{2} \\
1-x & : & \frac{1}{2} \leqslant x \leqslant 1 \\
x-1 & : & 1 \leqslant x<\infty
\end{array}\right.
$$

and $\eta$ be given as follows:

$$
\eta(y, x)= \begin{cases}y+x: \quad y \geqslant-\frac{1}{2} \\ 1: \quad y<-\frac{1}{2}\end{cases}
$$

Note that $x=1$ is a local minimum of $f$ and $\partial^{\eta} f(1)=\{v: v \leqslant-1 / 2\}$. Now we shall show that under certain assumptions the condition that $0 \in \partial^{\eta} f(x)$ where $x$ is a local minimum of $f$ becomes necessary.

Penot [4] has introduced the notion of a lower subdifferential $\partial_{l} f(x)$ for a function $f: R^{n} \rightarrow R$ at $x$ as follows:

$$
\partial_{l} f(x)=\left\{\xi \in R^{n}: f^{\prime}(x, v) \geqslant\langle\xi, v\rangle \text { for all } v \in R^{n}\right\}
$$

where $f^{\prime}(x, v)=\lim _{\lambda \rightarrow 0+}(f(x+\lambda v)-f(x)) / \lambda$ which is assumed to exist.

If $x \in R^{n}$ is a local minimum then $f^{\prime}(x, v) \geqslant 0$ for all $v \in R^{n}$ and hence the condition that $0 \in \partial_{l} f(x)$ turns out to be a necessary condition for a local minimum. We further note that when $f$ is convex, $\partial_{l} f(x)$ coincides with the subdifferential of convex analysis [6]. Following Penot, we now introduce the lower $\eta$-subdifferential of $f$ with respect to a given $\eta$ as follows:

$$
\partial_{l}^{\eta} f(x)=\left\{\xi \in R^{n} ; f^{\prime}(x, \eta(y, x)) \geqslant\langle\xi, \eta(y, x)\rangle \text { for all } y \in R^{n}\right\} .
$$

By definition, if $x$ is a local minimum of $f$, then $0 \in \partial_{l}^{\eta} f(x)$. Thus at a local minimum $\partial_{l}^{\eta} f(x) \neq \emptyset$. 
THEOREM 3.1. Let $f: R^{n} \rightarrow R$ be a semi-invex function with respect to a given $\eta$ satisfying Condition $C$. Let $x_{0} \in R^{n}$ be a local minimum of $f$. Suppose that $f^{\prime}\left(x_{0}, \eta\left(y, x_{0}\right)\right)$ exists for all $y \in R^{n}$. Then $0 \in \partial^{\eta} f\left(x_{0}\right)$.

Proof: Since $x_{0}$ is a local minimum, $0 \in \partial_{l}^{\eta} f(x)$. It is enough to show that $\partial_{l}^{\eta} f\left(x_{0}\right) \subseteq \partial^{\eta} f\left(x_{0}\right)$. By Proposition 2.4, $f$ is pre-invex with the same $\eta$. Hence for all $\lambda \in(0,1)$

$$
\begin{aligned}
f\left(x_{0}+\lambda \eta\left(x, x_{0}\right)\right) & \leqslant \lambda f(x)+(1-\lambda) f\left(x_{0}\right) \\
\frac{f\left(x_{0}+\lambda \eta\left(x, x_{0}\right)\right)-f\left(x_{0}\right)}{\lambda} & \leqslant f(x)-f\left(x_{0}\right) .
\end{aligned}
$$

Letting $\lambda \rightarrow 0+$, we have

$$
f^{\prime}\left(x_{0}, \eta\left(x, x_{0}\right)\right) \leqslant f(x)-f\left(x_{0}\right)
$$

If $\xi \in \partial_{l}^{\eta} f\left(x_{0}\right)$, it follows that

$$
f(x)-f\left(x_{0}\right) \geqslant\left\langle\xi, \eta\left(x, x_{o}\right)\right\rangle
$$

which implies that $\xi \in \partial^{\eta} f\left(x_{0}\right)$ and hence $\partial_{l}^{\eta}\left(x_{0}\right) \subseteq \partial^{\eta} f\left(x_{0}\right)$.

We note that if $f: R^{n} \rightarrow R$ is a locally Lipschitz function which is semi-invex at a local minimum $x_{0} \in R^{n}$ and if Assumption $\mathrm{A}$ is satisfied at $x_{0}$ then $f_{\eta}^{\circ}\left(x_{0}, h\right) \geqslant 0$ for all $h \in R^{n}$.

We next consider the following constrained minimisation problem $(\mathrm{P})$.

$$
\text { Minimise } f(x) \text { subject to } g_{i}(x) \leqslant 0, \quad i=1,2, \ldots, m \quad x \in R^{n} \text {. }
$$

where $f$ and the $g_{i}$ 's are scalar-valued locally Lipschitz functions which are semi-invex with the same $\eta$. It is well known [1] that if $f$ and the $g_{i}$ 's are locally Lipschitz, then the Generalised Kuhn-Tucker conditions, involving Clarke's subdifferential, will necessarily follow. But these conditions are not sufficient even if the functions are assumed to be semi-invex since Clarke's subdifferential in general differs from the $\eta$-subdifferential. But conditions involving the $\eta$-subdifferential can be established with are sufficient for optimality.

Let $A_{0}$ denote the set of feasible solutions for the problem (P).

ThEOREM 3.2. Consider (P), where $f$ and the $g_{i}$ 's are semi-invex with the same $\eta: R^{n} \times R^{n} \rightarrow R^{n}$. Let $x_{0} \in A_{0}$. Then $x_{0}$ is an optimal solution to $(P)$ if there exists $\lambda \in R_{+}^{n}$ such that

$$
\begin{aligned}
& \text { (i) } 0 \in \partial^{n} f\left(x_{0}\right)+\sum_{i=1}^{m} \lambda_{i} \partial^{\eta} g_{i}\left(x_{0}\right) \\
& \text { (ii) } \lambda_{i} g_{i}\left(x_{0}\right)=0 \text { for all } i=1,2, \ldots, m .
\end{aligned}
$$


Proof: By (i) there exist $\alpha \in \partial^{\eta} f\left(x_{0}\right)$ and $\beta_{i} \in \partial^{\eta} g_{i}\left(x_{0}\right)$ such that

$$
0=\alpha+\sum_{i=1}^{m} \lambda_{i} \beta_{i}
$$

Therefore by the semi-invexity of the $g_{i}$ 's, we have for all $x \in A_{0}$,

$$
\sum_{i=1}^{m} \lambda_{i}\left(g_{i}(x)-g_{i}\left(x_{0}\right)\right) \geqslant\left\langle-\alpha, \eta\left(x, x_{0}\right)\right\rangle .
$$

From (ii),

$$
0 \geqslant \sum_{i=1}^{m} \lambda_{i} g_{i}(x) \geqslant\left\langle-\alpha, \eta\left(x, x_{0}\right)\right\rangle
$$

Now the result follows by the semi-invexity of $f$.

We note that the conditions given here are not in general necessary. For example, consider the problem, Min $f(x)$, subject to $g(x) \leqslant 0$, where $f(x)$ and $g(x)$ are as in Example 2.2. Here $x=0$ is the optimum but $0 \notin \partial^{\eta} f(0)+\lambda \partial^{\eta} g(0)$, where $\lambda>0$.

\section{REFERENCES}

[1] F.H. Clarke, Optimization and nonsmooth analysis (Wiley Interscience, New York, 1983).

[2] B.D. Craven, 'Nondifferentiable optimization by smooth approximation', Optimization 17 (1986), 3-17.

[3] M.A. Hanson, 'On the sufficiency of Kuhn-Tucker conditions', J. Math. Anal. Appl. 80 (1981), 545-550.

[4] S. Mohan and S. Neogy, 'On invex sets and pre-invex functions', J. Math. Anal. Appl. 189 (1995), 901-908.

[5] J. Penot, 'Calcul sous-differentiel et optimisation', J. Funct. Anal. 27 (1978), 248-276.

[6] R. Pini, 'Invexity and generalised convexity', Optimization 22 (1991), 513-525.

[7] R.T. Rockafellar, Convex analysis (Princeton University Press, Princeton, NJ, 1970).

[8] X.Q. Yang and B.D. Craven, 'Necessary optimality conditions with modified subdifferential', Optimization 22 (1991), 387-400. 